\title{
Epicardial and Perihepatic Fat as Cardiometabolic Risk Predictors in Girls with Turner Syndrome: A Cardiac Magnetic Resonance Study
}

\author{
(D) Nanees A. Salem¹, (D) Nihal M. Batouty², (D) Ahmed M. Tawfik², (D) Donia M. Sobh², (D) Basma Gadelhak2, (D) Shimaa R. Hendawy33, \\ (D) Wafaa Laimon 1
}

\author{
1 Mansoura University Faculty of Medicine, Department of Pediatrics, Unit of Pediatric Endocrinology and Diabetes, Dakahlia, Egypt \\ 2 Mansoura University Faculty of Medicine, Department of Diagnostic and Interventional Radiology, Dakahlia, Egypt \\ ${ }^{3}$ Mansoura University Faculty of Medicine, Department of Clinical Pathology, Dakahlia, Egypt
}

\section{What is already known on this topic?}

Turner syndrome (TS) patients are at high risk of cardiometabolic disorders.

\section{What this study adds?}

TS girls displayed adverse cardiometabolic profile during late childhood and adolescence. Cardiac magnetic resonance-derived epicardial fat-thickness (EFT) and perihepatic fat-thickness are emerging cardiometabolic risk predictors in TS patients. Excess EFT, rather than total body adiposity, may contribute to altered metabolic profile among lean patients with TS.

\section{Abstract}

Objective: Turner syndrome (TS) patients are at high risk of cardiometabolic disorders. Cardiometabolic risk factors are more commonly related to visceral rather than total body adiposity. Adipocytokines have been explored as a potential link between obesity and obesityrelated cardiometabolic dysfunction. This study explored the validity of epicardial fat-thickness (EFT) and perihepatic fat-thickness (PHFT) measurement as cardiometabolic-risk predictors in TS-girls in relation to standard obesity-indices and metabolic syndrome (MetS) components.

Methods: Forty-six TS girls and twenty-five controls (10-16 years) were subdivided into two age-groups (10 to less than 13 and 13 16). Participants were assessed for body mass index (BMI) Z-scores, waist circumference (WC), total-fat mass (FM) and trunk-FM by bioimpedance-technique, EFT and PHFT by cardiovascular magnetic resonance, lipid-profile, homeostasis model assessment of insulin resistance (HOMA-IR), and serum chemerin. MetS was defined according to International Diabetes Federation criteria.

Results: Overweight/obesity and MetS were detected in $45.7 \%$ and $37 \%$ of TS-girls respectively. BMI Z-Score, WC, total-FM, trunk-FM, EFT and PHFT values were significantly higher in TS-age groups compared to age-matched control groups, being more pronounced in the older group when TS-girls had been exposed to estrogen. Dyslipidemia, higher HOMA-IR, chemerin, EFT and PHFT values were observed in lean-Turner compared to BMI-Z-matched controls. EFT and PHFT were significantly correlated with chemerin and several components of MetS. EFT at a cut-off-value of $6.20 \mathrm{~mm}$ (area under the curve $=0.814$ ) can predict MetS in TS-girls.

Conclusion: TS-girls displayed an adverse cardiometabolic profile during late childhood and adolescence. EFT and PHFT are emerging cardiometabolic risk predictors in TS-patients. Excess EFT rather than total body adiposity may contribute to altered metabolic profile among lean-Turner patients.

Keywords: Epicardial fat, perihepatic fat, metabolic syndrome, Turner syndrome

Address for Correspondence: Nanees A. Salem, MD, Mansoura University Faculty of Medicine, Department of Pediatrics, Unit of Pediatric Endocrinology and Diabetes, Dakahlia, Egypt

Phone: +201007553665 E-mail: nanees.salem@gmail.com ORCID: orcid.org/0000-0001-6783-9095

${ }^{\circ}$ Copyright 2021 by Turkish Pediatric Endocrinology and Diabetes Society

The Journal of Clinical Research in Pediatric Endocrinology published by Galenos Publishing House.
Conflict of interest: None declared Received: 28.01.2021 Accepted: 19.05.2021 


\section{Introduction}

Childhood obesity represents a major public-health crisis that has persistently increased in incidence throughout recent decades at an alarming rate (1). Metabolic syndrome (MetS) is a clustering of co-incident cardiometabolic risk factors, which predict adverse cardiovascular outcomes in adulthood (2).

Turner syndrome (TS) is one of the most common chromosomal disorders in females caused by complete or partial deficit of the second X-chromosome (3). Current epidemiological evidence indicates that children and adolescents with TS are susceptible to a wide spectrum of cardiometabolic risk factors compared to age-matched controls including: higher obesity-indices; impaired glucose metabolism; and atherogenic lipid profile $(4,5,6,7)$.

According to the adipose tissue expandability hypothesis, excess visceral fat (within/or surrounding viscera) together with relatively less subcutaneous adipose tissue, elicit a state of chronic low-grade inflammation that increases the risk of cardiovascular diseases (8). Currently, visceral fat deposition has been identified as an emerging marker of cardiovascular risk (9). Epicardial adipose tissue, the visceral fat reservoir of the heart, is enclosed between the pericardium and the myocardium layers and secrets several adipocytokines, some of which are mediators of inflammation (10).

Cardiovascular magnetic resonance (CMR) imaging is considered the standard reference for epicardial adipose tissue quantification (11). Epicardial fat thickness (EFT) shows good correlation with visceral abdominal fat, components of MetS, and severity of cardiovascular diseases $(12,13)$.

Chemerin, is an adipocytokine that modulates glucose and lipid metabolism in adipocytes (14). Chimerin has displayed strong associations with MetS components (15), and with EFT in adults with coronary artery disease (16). Thus, it may form an integral link between obesity and obesity-related cardiometabolic dysfunction (17).

Therefore, the aim was to explore for the first time the validity of EFT and perihepatic fat thickness (PHFT) as cardiometabolic risk predictors in girls with TS in relation to standard obesity-indices and components of MetS.

\section{Methods}

This case-control study included forty-six girls with TS (age range: 10-16 years) and twenty-five age-matched healthy girls. Girls with TS were recruited sequentially between September 2018 and November 2019 during their routine visits to the Pediatric Endocrinology Clinic at Mansoura
University Children's Hospital. The study was approved by the Ethics Committee of Mansoura Faculty of MedicineInstitutional Research Board (code no. R.20.04.800). Informed consent was obtained from the parents of all participants included in the study.

Girls with TS follow a uniform protocol for recombinant human growth hormone (rGH) therapy; thirty-six girls were receiving $\mathrm{rGH}(0.05 \mathrm{mg} / \mathrm{kg} /$ day $)$ while ten girls in the cohort had stopped rGH at a mean age of $14.4 \pm 0.8$ year as height velocity $<3 \mathrm{~cm} / y e a r$. Estrogen replacement therapy (ERT), oral ethinylestradiol at initial dose of $2 \mathrm{mg} /$ day, was initiated for girls who had exhibited no clinical signs of spontaneous puberty by 14 years. Seven girls had spontaneous puberty at a mean age of $13.9 \pm 0.4$ years. TS girls having chronic comorbidities, thyroid dysfunction, congenital/acquired heart diseases or currently receiving medications, other than rGH and/or ERT, were excluded from the study.

\section{Clinical Evaluation}

Anthropometric measurements, including weight, height, and waist circumference (WC), were obtained by a trained nurse according to standardized techniques. Body mass index (BMI) was calculated as weight divided by height squared $\left(\mathrm{kg} / \mathrm{m}^{2}\right)$. Height and BMI Z-scores were calculated using reference data for Egyptian children and adolescents (18). In girls with TS, BMI Z-score was corrected for patient's height age to adjust for the effect of short stature (19). Girls with TS were classified based on WHO BMI Z-score cut-offs into a "lean-group" [BMI Z-score $\leq+1$ standard deviation (SD)] or an "overweight/obese-group" (BMI Z-score > +1 SD) (20). Systolic and diastolic blood pressure (SBP/DBP) was measured by standard technique (21).

Participants were classified according to Tanner breast scale into pre-pubertal (stage 1), early-puberty (stages 2-3) and late-puberty (stages 4-5). Girls with TS were subdivided into two groups; pre-pubertal (10-to less than 13 years) and pubertal (13-16 years) groups, the latter group included both early-and late-puberty stages. The controls were subdivided into two groups; early-puberty (10-to less than 13 years) and late-puberty (13-16 years) groups.

\section{Definition of Metabolic Syndrome}

In the TS group, MetS was diagnosed according to the 2007 International Diabetes Federation (IDF) pediatric definition for MetS (2), with the exception of blood pressure for which "elevated blood pressure" was defined according to the 2017 Clinical Practice Guideline for Screening and Management of High Blood Pressure in Children and Adolescents (21). MetS was diagnosed by central adiposity (WC $\geq 90^{\text {th }}$ percentile for age and gender) and the presence of at least two of the 
remaining four criteria which are: Fasting blood glucose $\geq 100 \mathrm{mg} / \mathrm{dL}$ ( $5.6 \mathrm{mmol} / \mathrm{L})$; triglycerides levels $\geq 150 \mathrm{mg} / \mathrm{dL}$ $(1.7 \mathrm{mmol} / \mathrm{L})$; HDL-c level $\leq 40 \mathrm{mg} / \mathrm{dL}(1.03 \mathrm{mmol} / \mathrm{L})$; and SBP and/or DBP $\geq 90^{\text {th }}$ percentile for age, gender and height percentile.

\section{Body Fat Composition Evaluation}

Total-fat mass (FM; kg) and trunk-FM (kg), a marker of central (abdominal) adiposity, were measured by a bioimpedance technique using a Tanita BC-418MA body composition analyzer (Tanita Corp., Tokyo, Japan).

\section{Biochemical Evaluation}

Blood samples were collected in the morning, after a 12hour overnight fast. Sera were stored at $-20{ }^{\circ} \mathrm{C}$. Total cholesterol and triglycerides were measured by colorometric kit (Spinreact, Girona, Spain), and HDL-c was measured by colorometric kit (Human Diagnostics, Wiesbaden, Germany). Serum chemerin $(\mathrm{ng} / \mathrm{mL})$ was measured using an enzymelinked immunosorbent assay-Sandwich technique (SUN RED, Shanghai, China; cat. no. 201-12-1436). Homeostasis model assessment of insulin resistance (HOMA-IR) was calculated as FBG $(\mathrm{mg} / \mathrm{dL}) \times$ fasting insulin $(\mathrm{mIU} / \mathrm{L}) / 405$.

\section{Cardiac Magnetic Resonance Imaging}

The measurements of EFT and PHFT were performed by a single expert technician who was blinded to the study groups. CMR was performed using a $1.5 \mathrm{~T}$ Tesla MR imaging (MRI) machine (Ingenia, Philips, Netherland). Electrocardiogram gated cine steady state free precession (SSFP) images were acquired in short axis-view (SA) (slice thickness $5 \mathrm{~mm}$, slice gap $-1 \mathrm{~mm}, \mathrm{TR}=3.2 \mathrm{~ms}, \mathrm{TE}=1.6$ $\mathrm{ms}$, matrix 175/352, FOV $=350 \mathrm{~mm}^{2}$, slices 25), while modified Dixon (mDixon) sequence was obtained in SA plane (slice thickness $5 \mathrm{~mm}$, slice gap $-2.5 \mathrm{~mm}$, TR $=5.9$ $\mathrm{ms}, \mathrm{TE}=0.0 \mathrm{~ms}$, matrix $151 / 320, \mathrm{FOV}=400 \mathrm{~mm}^{2}$, slices 92). Image analysis was performed by a single radiologist (N.B.) who was also blinded to the study groups. Images were transferred to workstation (extended MR Workspace 2.6.3.5, Philips medical systems, Netherland). Maximum EFT was measured opposite the right ventricular free wall in the following sequences; m-Dixon (SA-view) and cine SSFP (SA-view) at end systole and end diastole. Maximum PHFT was measured in sub-diaphragmatic region during cine SSFP (SA-view) (Supplementary Figure 1).

\section{Statistical Analysis}

Data were analyzed using Statistical Package for the Social Sciences, version 23.0 (IBM Inc., Chicago, IL, USA). Categorical data are presented as number and percent, and chi-square test or Fisher exact test were used as appropriate for comparison of two groups. Continuous data were tested for normality using one-sample Kolmogorov-Smirnov test, then data presented as mean \pm SD for parametric data and median (minimum-maximum) for non-parametric data. Two groups were compared using Student's t-test for parametric data and Mann-Whitney $U$ test for non-parametric data. Pearson and Spearman correlation analysis were used to correlate parametric and non-parametric data respectively. Receiver operating characteristic (ROC) curves were constructed to analyze the discriminative power of EFT, and PHFT to predict MetS among girls with TS. Areas under curves (AUCs) and 95\% confidence interval, optimal cutoff-values with relevant specificity, sensitivity, and accuracy were determined. Statistical significance was set at $p<0.05$.

\section{Results}

The study included two age-matched groups, Turnergroup $(n=46)$ with a mean age of $13.14 \pm 3.15$ years and a control-group $(n=25)$ with a mean age of $12.17 \pm 3.02$. Based on karyotype results, two groups were identified; 45 , $X$ group $(n=24)$ and non-45, $X$ group $(n=22)$, the latter group including isochromosome 46,X,i[Xq10] $(n=12)$, deletion 46,X,del(Xp-) $(n=4)$, and different forms of mosaicism $(n=6)$. No significant differences were detected in cardiometabolic variables between 45, $\mathrm{X}$ and non-45, $\mathrm{X}$ groups ( $p>0.05)$ (Supplementary Table 1).

Girls with TS in the pre-pubertal (10-13 years) group displayed significantly higher total-FM, trunk-FM, serum cholesterol, HOMA-IR and EFT values compared to agematched controls. Girls with TS in the pubertal (13-16 years) group displayed similar significant differences to the prepubertal group with the addition of significantly higher BMI Z-score, WC, serum triglycerides, and PHFT values compared to age-matched control groups (Table 1).

Based on BMI status, the overall prevalence of overweight/ obesity in girls with TS was $45.7 \%$ (21/46). When the age groupings were considered separately this was 8/26 $(30.8 \%)$ in the pre-pubertal group and 13/20 (65\%) within pubertal group. Overweight/obese Turner-group were significantly older and had significantly higher BMI Z-score, WC, total-FM, trunk-FM, HOMA-IR, serum cholesterol and triglycerides, EFT and PHFT values compared to both leanTurner and control groups. Interestingly, lean-Turner had significantly higher HOMA-IR, serum cholesterol, and EFT values compared to controls, although BMI Z-score, totalFM, and trunk-FM were significantly lower in lean-Turner compared to age-matched controls (Table 2). 
Based on IDF criteria (2), the prevalence of MetS in TS girls was $37 \%$ (17/46). Girls with TS were then sub-classified into a "MetS-group" ( $n=17)$ and a "non-MetS group" $(n=29)$ and compared. All girls in the MetS-group were obese, while in the non-MetS group, twelve girls $(41.4 \%)$ were overweight/ obese. BMI Z-score, WC, total-FM, trunk-FM, HOMA-IR, cholesterol, triglycerides, EFT and PHFT were significantly higher in the MetS-group compared to the non-MetS and control groups. Although non-MetS and control groups were matched for BMI Z-score and WC, non-MetS group displayed significantly higher total-FM, trunk-FM, HOMA-IR, cholesterol and EFT compared to control girls (Table 3).

Serum chemerin levels were significantly higher in TS participants compared to age-matched control-groups; in overweight/obese-Turner compared to lean-Turner $(p=0.014)$ and control $(p<0.001)$ groups; in the MetSgroup compared to the non-MetS $(p=0.044)$ and control $(p<0.001)$ groups; and in non-MetS and lean-Turner groups compared to controls. Serum chemerin was positively correlated with age, BMI Z-score, WC, total-FM, trunk-FM, HOMA-IR, triglycerides, EFT and PHFT in TS (Table 4). However, these correlations were not evident in the control group (Supplementary Table 2).
EFT and PHFT were positively correlated, EFT at different sequences including; m-Dixon (SA-view) and cine SSFP (SA-view) at end systole and end diastole were positively correlated with age, BMI Z-score, WC, HOMA-IR, triglycerides, total-FM, and trunk-FM, while PHFT showed similar correlations but was not correlated with triglycerides (Table 4).

Regarding prediction of MetS among TS girls, EFT in mDixon SA-view with a cut-off-value of $6.20 \mathrm{~mm}$ had the highest discriminative power among CMR-derived parameters $\quad(A U C=0.814) \quad(84.6 \% \quad$ sensitivity; $\quad 73.5 \%$ specificity), while PHFT with a cut-off-value of $5.15 \mathrm{~mm}$ had the lowest discriminative power (AUC $=0.685$ ). AUC of EFT at mDixon SA-view was comparable to those of standard cardiometabolic risk factors; BMI Z-score (AUC $=0.998)$, WC (AUC $=0.955)$, HOMA-IR (AUC $=0.899)$, and triglycerides $(A U C=0.885)$. Finally, serum chemerin of more than 250 $\mathrm{ng} / \mathrm{mL}$ can predict MetS in girls with TS with $\mathrm{AUC}=0.834$, $76.9 \%$ sensitivity and $77.6 \%$ specificity (Table 5 and Figure 1a, 1b).

Interestingly, Supplementary Figure 1 represents CRM imaging of a lean Turner girl aged 15 years and 2 months (height age-adjusted BMI Z-score $=0.9$ ), although lean, the

Table 1. Clinical, body composition, biochemical and cardiovascular magnetic resonance parameters among Turner syndrome and control age-groups

\begin{tabular}{|c|c|c|c|c|c|c|}
\hline & \multicolumn{2}{|l|}{ Turner group $(n=46)$} & \multicolumn{2}{|c|}{ Control group $(n=25)$} & \multicolumn{2}{|c|}{ Test of significance } \\
\hline & $\begin{array}{l}10-13 \text { years } \\
(n=26)\end{array}$ & $\begin{array}{l}13-16 \text { years } \\
(n=20)\end{array}$ & $\begin{array}{l}10-13 \text { years } \\
(n=10)\end{array}$ & $\begin{array}{l}13-16 \text { years } \\
(n=15)\end{array}$ & $\mathrm{p} 1$ & $\mathrm{p} 2$ \\
\hline \multicolumn{7}{|l|}{ Clinical parameters } \\
\hline Median BMI Z-score & $0.43(-1.1,4)$ & $1.5(-1.5,3.1)$ & $0.9(-0.6,1.0)$ & $0.7(0.0,1.0)$ & 0.549 & $0.031^{*}$ \\
\hline Mean WC (cm) & $66.69 \pm 8.82$ & $76.75 \pm 7.79$ & $66.54 \pm 5.51$ & $72.71 \pm 2.69$ & 0.960 & $0.042 *$ \\
\hline \multicolumn{7}{|l|}{ Body composition parameters } \\
\hline Median total body FM (kg) & $7.80(5.5-17.4)$ & $13.45(7.2-28.9)$ & $5.10(3.43-8.6)$ & $10.0(5.4-13.8)$ & $0.022 *$ & $0.019 *$ \\
\hline Median trunk FM (Kg) & $3.20(2.5-7.9)$ & $7.15(3.4-15.9)$ & $2.20(0.7-3.8)$ & $4.20(0.6-6.5)$ & $0.010 *$ & $0.025 *$ \\
\hline \multicolumn{7}{|l|}{ Biochemical parameters } \\
\hline Median HOMA-IR & $2.11(0.96-5.43)$ & $4.27(0.92-8.26)$ & $1.66(0.79-2.01)$ & $1.48(1.06-2.23)$ & $0.047 *$ & $0.001 *$ \\
\hline Mean cholesterol (mg/dL) & $145.6 \pm 26.7$ & $176.8 \pm 35.5$ & $121.5 \pm 9.5$ & $136.3 \pm 11.1$ & $0.011^{*}$ & $<0.001^{*}$ \\
\hline Mean triglycerides (mg/dL) & $95.5 \pm 27.5$ & $111.8 \pm 40.5$ & $80.4 \pm 10.7$ & $78.1 \pm 13.8$ & 0.088 & 0.041 * \\
\hline Mean HDL (mg/dL) & $54.61 \pm 13.73$ & $55.66 \pm 13.07$ & $56.83 \pm 11.68$ & $49.57 \pm 5.25$ & 0.139 & 0.242 \\
\hline Median chemerin (ng/mL) & $249.4(108.5-388.5)$ & $353.5(109.3-630.3)$ & $128.5(35.5-136)$ & $131.0(119-175)$ & $0.033^{*}$ & $0.008 *$ \\
\hline \multicolumn{7}{|l|}{ CMR parameters } \\
\hline Mean EFT-SA mDixon (mm) & $5.26 \pm 1.65$ & $7.64 \pm 1.80$ & $4.68 \pm 0.51$ & $5.18 \pm 1.17$ & 0.294 & $0.002 *$ \\
\hline Mean EFT-SA systole (mm) & $6.35 \pm 1.64$ & $9.19 \pm 2.04$ & $4.55 \pm 0.51$ & $5.17 \pm 0.63$ & $0.002 *$ & $<0.001^{*}$ \\
\hline Mean EFT-SA diastole (mm) & $3.79 \pm 1.14$ & $4.89 \pm 1.61$ & $2.38 \pm 0.31$ & $2.94 \pm 0.47$ & $0.001 *$ & $0.004 *$ \\
\hline Mean PHFT (mm) & $5.62 \pm 1.86$ & $6.31 \pm 2.37$ & $4.57 \pm 0.38$ & $4.99 \pm 1.06$ & 0.134 & $0.023 *$ \\
\hline \multicolumn{7}{|c|}{$\begin{array}{l}\text { p1: Turner group vs. control group (10-13 years); p2: Turner group vs. control group (13-16 years). } \\
\text { BMI: body mass index, CMR: cardiovascular magnetic resonance, EFT: epicardial fat thickness, FM: fat mass, HOMA-IR: homeostasis model assessment of insulin } \\
\text { resistance, HDL: high density lipoprotein, SA: short axis view, PHFT: perihepatic fat thickness, WC: waist circumference, SD: standard deviation }\end{array}$} \\
\hline
\end{tabular}


results of EFT in different sequences and PHFT exceeded the established cut-off values derived from ROC analysis (Table 5).

\section{Discussion}

In the current study and for the first time, the clinical relevance of CMR-derived EFT and PHFT as cardiometabolic risk predictors in girls with TS in relation to standard obesityindices and components of MetS was investigated.

Data on the prevalence of MetS in pediatric and adult TS cohorts are limited. In the current study, the prevalence of overweight/obesity and MetS were $45.7 \%$ (21/46), and $37 \%$ (17/46) respectively. In adult TS, Calcaterra et al (22) reported a prevalence of MetS of $4.7 \%$ (4/85), whereas Álvarez-Nava et al (23) reported a prevalence of overweight/obesity and MetS to be $40 \%$ (35/88) and $49 \%$ (43/88), respectively. In a pediatric TS cohort $(n=19)$, O'Gorman et al (5) found that 7/19 girls met one criterion for MetS, 8/19 met two criteria, but none fulfilled the diagnosis of MetS. The discrepancy in the prevalence of MetS may mostly be related to ethnic differences in rates of obesity and MetS components and different criteria used to define MetS. In the current study it was observed that, although Turner girls in the non-MetS group were age, BMI Z-score and WC-matched with control girls, they had significantly higher total-FM, trunk-FM, HOMA-IR, total cholesterol and EFT values. These findings can be explained because $41.4 \%$ of girls in the non-MetS group were overweight/obese.

However, although BMI Z-score, total-FM, and trunkFM values were significantly higher in the control group compared to lean-Turner, interestingly, the lean-Turner group had significantly higher HOMA-IR, total cholesterol, and EFT values compared to control girls, together with the significant associations between EFT and PHFT and triglycerides and HOMA-IR. These observations support the utility of visceral adipose tissue deposition measurement from traditional measurements of body adiposity, and also highlight that visceral fat, rather than subcutaneous adipose tissue, is more metabolically active and has a key role in the development of different cardiometabolic risk factors (8).

Similar associations between EFT and cardiometabolic risk factors and with increased carotid intima-media thickness

Table 2. Clinical, body composition, biochemical and cardiovascular magnetic resonance parameters in girls with Turner syndrome according to BMI status compared to control group

\begin{tabular}{|c|c|c|c|c|c|c|}
\hline & \multicolumn{2}{|l|}{ Turner group $(n=46)$} & \multirow{2}{*}{$\begin{array}{l}\text { Control group } \\
(n=25)\end{array}$} & \multicolumn{3}{|c|}{ Test of significance } \\
\hline & $\begin{array}{l}\text { Overweight/obese } \\
(n=21)\end{array}$ & Lean $(n=25)$ & & $\mathrm{p} 1$ & $\mathrm{p} 2$ & p3 \\
\hline \multicolumn{7}{|l|}{ Clinical parameters } \\
\hline Mean age (years) & $14.46 \pm 2.26$ & $12.04 \pm 3.41$ & $12.17 \pm 3.02$ & $0.007 *$ & 0.879 & $0.006 *$ \\
\hline Median height Z-score & $-3.65(-4.8--2.1)$ & $-2.89(-5.0--1.3)$ & $0.90(-1.5-1.7)$ & $<0.001 *$ & $<0.001$ * & 0.165 \\
\hline Median BMI Z-score & $2.15(1.1-4.0)$ & $-0.15(-1.7-1.0)$ & $0.70(0.0-1.0)$ & $<0.001 *$ & $<0.001$ * & $<0.001$ * \\
\hline Mean WC (cm) & $78.81 \pm 6.66$ & $63.72 \pm 7.96$ & $66.02 \pm 7.28$ & $<0.001 *$ & 0.292 & $<0.001$ * \\
\hline \multicolumn{7}{|l|}{ Body composition parameters } \\
\hline Median total body FM (kg) & $12.00(4.6-31.9)$ & $4.50(2.4-14.0)$ & $7.60(2.6-10.8)$ & $<0.001^{*}$ & $<0.001 *$ & $<0.001$ * \\
\hline Median trunk FM (kg) & $4.70(2.1-15.9)$ & $1.60(0.6-5.5)$ & $3.20(1.2-6.5)$ & $0.001 *$ & $<0.001$ * & $<0.001$ * \\
\hline \multicolumn{7}{|l|}{ Biochemical parameters } \\
\hline Median HOMA-IR & $4.15(1.82-8.26)$ & $2.29(0.92-4.71)$ & $1.48(0.35-2.23)$ & $<0.001 *$ & $0.001 *$ & $0.004 *$ \\
\hline Mean total cholesterol (mg/dL) & $185.33 \pm 39.33$ & $155.16 \pm 15.49$ & $117.04 \pm 10.35$ & $<0.001 *$ & $0.010^{*}$ & $0.007 *$ \\
\hline Mean triglycerides (mg/dL) & $116.52 \pm 35.79$ & $74.72 \pm 9.24$ & $80.04 \pm 10.97$ & $<0.001 *$ & 0.161 & $0.007 *$ \\
\hline Men HDL (mg/dL) & $52.90 \pm 14.07$ & $51.84 \pm 13.73$ & $51.80 \pm 10.11$ & 0.759 & 0.991 & 0.797 \\
\hline Median chemerin (ng/mL) & $344.90(109.3-630.3)$ & $221.70(45.0-290.1)$ & $119.00(20.4-175.0)$ & $<0.001 *$ & $<0.001$ * & $0.014^{*}$ \\
\hline \multicolumn{7}{|l|}{ CMR parameters } \\
\hline Mean EFT-SA mDixon (mm) & $7.24 \pm 2.04$ & $5.69 \pm 2.12$ & $4.64 \pm 0.97$ & $<0.001^{*}$ & 0.031 * & $0.016^{*}$ \\
\hline Mean EFT-SA systole (mm) & $8.71 \pm 2.73$ & $6.54 \pm 2.22$ & $4.58 \pm 0.77$ & $<0.001 *$ & $<0.001$ * & $0.005^{*}$ \\
\hline Mean EFT-SA diastole (mm) & $4.88 \pm 1.76$ & $3.27 \pm 1.05$ & $2.87 \pm 0.65$ & $<0.001^{*}$ & 0.053 & $0.013 *$ \\
\hline Mean PHFT (mm) & $6.97 \pm 2.66$ & $5.11 \pm 1.14$ & $4.99 \pm 1.06$ & $0.001 *$ & 0.702 & $0.003^{*}$ \\
\hline \multicolumn{7}{|c|}{$\begin{array}{l}\text { Data presented either as mean SD or median (minimum-maximum). } \\
\text { *Significant difference }(\mathrm{p}<0.05) \text {. } \\
\text { p1: overweight/obese TS vs. control; p2: Lean TS vs. control; p3: overweight/obese TS vs. Lean TS. } \\
\text { BMI: body mass index, CMR: cardiovascular magnetic resonance, EFT: epicardial fat thickness, FM: fat mass, HOMA-IR: Homeostasis model assessment of insulin } \\
\text { resistance, HDL: high density lipoprotein, SA: short axis view, PHFT: perihepatic fat thickness, WC: waist circumference, SD: standard deviation }\end{array}$} \\
\hline
\end{tabular}



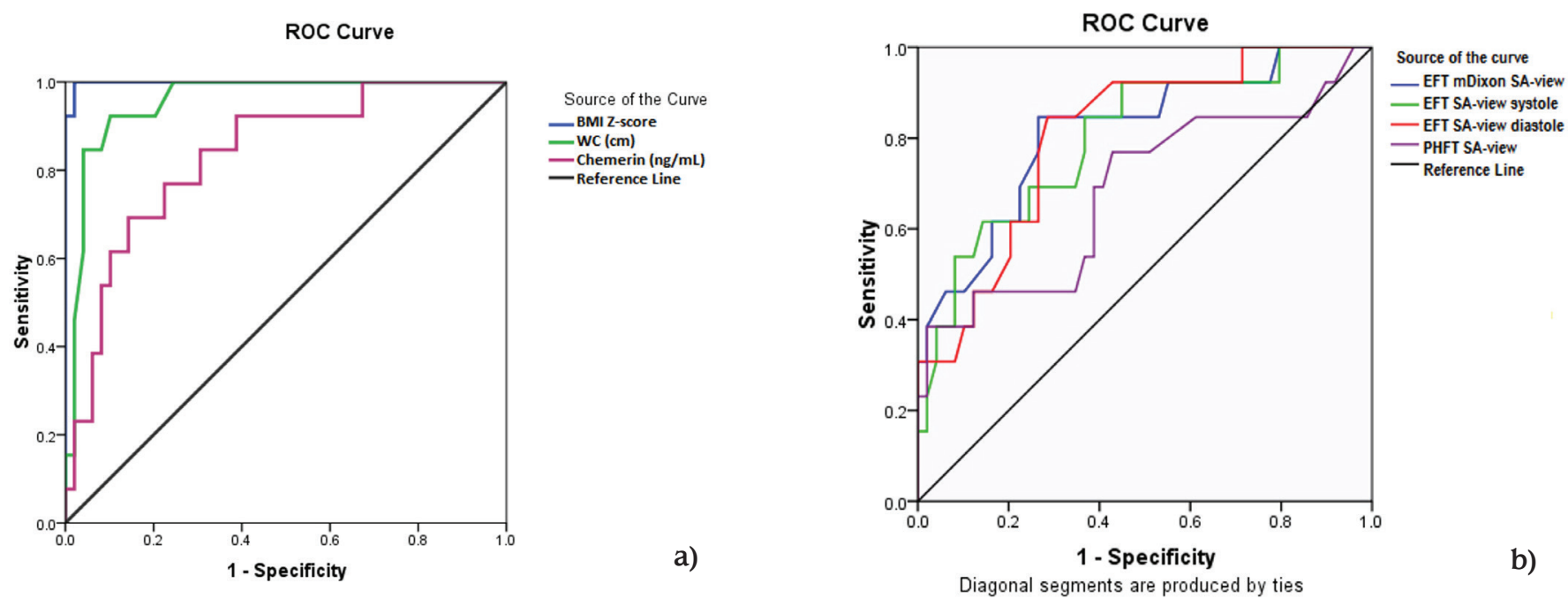

Figure 1. a) Receiver operating characteristic (ROC) curve for prediction of metabolic syndrome from BMI Z-score, WC and serum chemerin in girls with Turner syndrome. b) ROC curve for prediction of metabolic syndrome from epicardial fat thickness sequences and perihepatic fat thickness in girls with Turner syndrome

BMI: body mass index, WC: waist circumference, ROC: receiver operating characteristic, EFT: epicardial fat thickness, SA: short axis view

in obese children and adolescents were reported $(24,25,26)$.

In previous studies conducted among TS cohorts, O'Gorman et al (5) have demonstrated a significant increase in MRIderived subcutaneous adipose tissue with no significant differences in MRI-derived intra-myocellular lipid measured by MRI or in bioimpedance-derived body-FM between young TS girls and age-and BMI Z-score-matched controls, while Ostberg et al (27) demonstrated increased intrahepatocellular lipids using MRI in adult TS cohorts.

ROC analysis to assess the validity of EFT and PHFT in prediction of MetS in girls with TS revealed that EFT measured in an mDixon SA-view with a cut-off-value of $6.20 \mathrm{~mm}$ had the highest discriminative power $(\mathrm{AUC}=0.814)$, while PHFT had the lowest discriminative power ( $A U C=0.685$ ) among CMR-derived parameters. The results of EFT aproached those of standard cardiometabolic risk factors and also that of serum chemerin $(A U C=0.834)$.

Currently, there is no consensus for EFT cut-off values and most previous studies yielded different EFT cut-off values, mostly related to differences in samples, such as age, ethnicity and degree of obesity. Abaci et al (28) reported an EFT cut-off value of $4.1 \mathrm{~mm}$ for prediction of IR ( $90 \%$ sensitivity and $61 \%$ specificity), while Okyay et al (29) reported an EFT cut-off value of $4.35 \mathrm{~mm}$ for prediction of MetS (61.7\% sensitivity; $79.2 \%$ specificity) in obese children. However, there is almost no data available concerning PHFT measurements and no reported specific cut-off value for PHFT in the literature.

Interestingly, Supplementary Figure 1 shows the CRM imaging of a lean Turner girl aged 15 years and two months with a height/age-adjusted BMI Z-score of 0.9. Although she was lean, the results of the EFT at different sequences and PHFT exceeded the established cut-off values derived from ROC analysis in the current study.

Currently, two opposite phenotypes have been reported which are the "metabolically healthy obese" and the "metabolically obese but normal-weight" (30). The underlying mechanism of such apparent dissociation is not fully understood. However, advances in non-invasive imaging techniques are making significant inroads, allowing understanding of the fundamental contribution of visceral adiposity and fat distribution in such phenotypes, which potentially mediate their metabolic effects through adipocytokine production. Therefore, excess EFT rather than total-FM may explain the altered metabolic profile among the lean-Turner group with a potential role of chemerin as an adipocytokine.

The results of the current study revealed higher serum chemerin levels in overweight/obese-Turner compared to lean-Turner and control groups, and also in the MetS-group compared to non-MetS and control groups. It is worth noting that serum chemerin was significantly higher in non-MetS than in controls, despite being matched for age, BMI Z-scores, and WC. This could be due to $41.4 \%$ of girls in the non-MetS group being overweight/obese. However, serum chemerin levels were significantly higher in leanTurner than control girls, despite control girls having higher total-FM, and trunk-FM. The significantly increased EFT and PHFT in lean-Turner compared to control group together with significant correlation between chemerin and EFT and PHFT may point to the greater contribution of visceral fat 
(EF and PHF) rather than subcutaneous fat as a source of circulating chemerin.

In addition, positive correlations between chemerin and BMI Z-score, WC, FM, HOMA-IR, triglycerides, EFT, and PHFT were evident in the TS group but not in the control group, supporting the possible role of chemerin in mediating metabolic derangement in TS patients and indicating that serum chemerin may have increased in association with a pathological increase in body mass and excess body adiposity. Similarly, in previous studies, serum chemerin displayed strong associations with components of MetS (15), and with EF-volume (16). Thus, chemerin may form a pivotal link between obesity and obesity-related cardiometabolic dysfunction (17). In a recent study, chemerin levels were significantly increased in girls with TS compared with ageand BMI-matched controls but was not correlated with age, BMI Z-score, or any of the metabolic parameters including; fasting blood glucose, fasting insulin, HOMA-IR, triglyceride, and non-HDL (31).

In the current study, young TS patients displayed increased risk for overweight/obesity and for adverse cardiometabolic profile, whereas the metabolic derangements (high cholesterol and HOMA-IR), unfavorable body composition (increased total-FM and trunk-FM) and increased visceral adiposity (EFT) start to be evident in girls with TS in prepubertal (10-13 years) group, while older TS girls in the pubertal (13-16 years) group who had been eventually exposed to estrogen displayed similar abnormalities in addition to higher BMI Z-scores, WC, serum triglycerides, and PHFT values compared to age-matched control groups. Similarly, in a recent longitudinal study, metabolic comorbidities were found to start in childhood, increasing the risk for cardiovascular disease across the Turner patient's lifespan (7). These findings reinforce the importance of annual screening for cardiometabolic risk factors and early counseling regarding healthy nutrition and active lifestyle in young TS girls (3).

\section{Study Limitations}

The small sample size of the current TS cohort precluded reliable evaluation of cardiometabolic profile, EFT and PHFT of different karyotypes groups and the cross-sectional design precluded the evaluation of the beneficial/or adverse effects of rGH and ERT in the context of cardiometabolic

Table 3. Clinical, body composition, biochemical and cardiovascular magnetic resonance parameters among Turner syndrome subgroups (with and without metabolic syndrome) and control group

\begin{tabular}{|c|c|c|c|c|c|c|}
\hline & \multicolumn{2}{|c|}{ Turner syndrome $(\mathrm{n}=46)$} & \multirow[t]{2}{*}{ Control $(n=25)$} & \multicolumn{3}{|c|}{ Test of significance } \\
\hline & MetS group $(n=17)$ & $\begin{array}{l}\text { Non-MetS group } \\
(\mathrm{n}=29)\end{array}$ & & $\mathrm{p} 1$ & $\mathrm{p} 2$ & p3 \\
\hline \multicolumn{7}{|l|}{ Clinical parameters } \\
\hline Mean age (years) & $15.26 \pm 1.77$ & $13.86 \pm 2.07$ & $13.45 \pm 2.04$ & 0.012 & 0.515 & 0.046 \\
\hline Median BMI Z-score & $2.53(1.9,4.0)$ & $0.54(-1.5,2.1)$ & $0.68(0.2,0.9)$ & $<0.001 *$ & 0.607 & $<0.001$ * \\
\hline Mean WC (cm) & $82.00 \pm 4.30$ & $68.46 \pm 7.85$ & $66.82 \pm 5.51$ & $<0.001 *$ & 0.867 & $<0.001 *$ \\
\hline Median total body FM (kg) & $13.70(11.2-31.9)$ & $5.35(3.4-14.0)$ & $4.80(2.6-5.1)$ & $<0.001^{*}$ & $0.017^{*}$ & $<0.001$ * \\
\hline Median trunk FM (kg) & $5.70(3.4-15.9)$ & $2.50(0.6-7.2)$ & $1.75(1.2-2.2)$ & $<0.001 *$ & $0.013^{*}$ & $<0.001 *$ \\
\hline \multicolumn{7}{|l|}{ Biochemical parameters } \\
\hline Median HOMA-IR & $5.81(2.35-8.26)$ & $3.13(0.92-4.31)$ & $1.48(0.35-2.23)$ & $<0.001 *$ & $<0.001^{*}$ & $0.003 *$ \\
\hline Mean total cholesterol (mg/dL) & $183.53 \pm 34.87$ & $154.50 \pm 18.97$ & $118.68 \pm 9.89$ & $<0.001 *$ & $<0.001 *$ & $0.029 *$ \\
\hline \multicolumn{7}{|l|}{ CMR parameters } \\
\hline Men EFT-SA mDixon (mm) & $7.87 \pm 2.07$ & $6.22 \pm 1.86$ & $4.90 \pm 0.82$ & $<0.001 *$ & 0.004 & $0.019 *$ \\
\hline Mean EFT-SA systole (mm) & $9.06 \pm 2.75$ & $6.72 \pm 1.98$ & $4.78 \pm 0.63$ & $<0.001 *$ & $<0.001 *$ & $0.042 *$ \\
\hline Mean EFT-SA diastole (mm) & $5.16 \pm 1.98$ & $4.14 \pm 1.13$ & $2.59 \pm 0.46$ & $0.001 *$ & $<0.001^{*}$ & 0.053 \\
\hline Mean PHFT (mm) & $7.31 \pm 3.03$ & $5.67 \pm 1.53$ & $5.37 \pm 0.89$ & $0.042 *$ & 0.452 & $0.034^{*}$ \\
\hline
\end{tabular}


profile, EFT and PHFT. Nevertheless, our study is the first to identify the validity of EFT and PHFT as cardiometabolic risk predictors in TS patients. Thus it is to be hoped that this study will provide a stimulus for future larger studies.

\section{Conclusion}

Girls with TS display adverse cardiometabolic profile during late childhood and adolescence. CMR-derived EFT and PHFT are emerging tools for assessment of cardiometabolic risk and for prediction of MetS in a high-risk population, such as TS patients. There is an evident need to establish specific cut-off values for EFT and PHFT and when this has been achieved, it will improve the subsequent utility of EFT and PHFT as screening tools and follow-up markers for cardiometabolic risk in high-risk populations, such as girls with TS.

\section{Ethics}

Ethics Committee Approval: The study was approved by the Ethics Committee of Mansoura Faculty of MedicineInstitutional Research Board (code no. R.20.04.800).

\begin{tabular}{|c|c|c|c|c|c|c|}
\hline & & Serum chemerin & PHFT & EFT-SA mDixon & EFT-SA systole & EFT-SA diastole \\
\hline \multirow{2}{*}{ Age } & $\mathrm{r}$ & 0.508 & 0.406 & 0.621 & 0.622 & 0.369 \\
\hline & $\mathrm{p}$ & $<0.001^{*}$ & $0.005^{*}$ & $<0.001 *$ & $<0.001 *$ & $0.012^{*}$ \\
\hline \multirow{2}{*}{ BMI Z-score } & $\mathrm{r}$ & 0.368 & 0.417 & 0.343 & 0.373 & 0.251 \\
\hline & $\mathrm{p}$ & $0.012^{*}$ & $0.004 *$ & $0.020^{*}$ & $0.011 *$ & 0.093 \\
\hline WC & $\mathrm{p}$ & $0.003^{*}$ & $<0.001$ * & $0.002^{*}$ & $<0.001 *$ & $0.018^{*}$ \\
\hline \multirow{2}{*}{ Total body FM } & $\mathrm{r}$ & 0.483 & 0.378 & 0.450 & 0.477 & 0.399 \\
\hline & $\mathrm{p}$ & 0.001 * & $0.010^{*}$ & $0.002 *$ & $0.001 *$ & $0.006^{*}$ \\
\hline \multirow{2}{*}{ Trunk FM } & $\mathrm{r}$ & 0.431 & 0.326 & 0.363 & 0.393 & 0.340 \\
\hline & $\mathrm{p}$ & $0.003^{*}$ & 0.027 * & $0.013^{*}$ & $0.007^{*}$ & $0.021 *$ \\
\hline Triglycerides & $\mathrm{p}$ & 0.011 * & 0.296 & $0.019 *$ & 0.072 & 0.127 \\
\hline \multirow{2}{*}{ Chemerin } & $\mathrm{r}$ & - & 0.448 & 0.535 & 0.443 & 0.394 \\
\hline & $\mathrm{p}$ & - & $0.002 *$ & $<0.001 *$ & $0.002 *$ & $0.007^{*}$ \\
\hline \multirow{2}{*}{ PHFT } & r & - & - & 0.494 & 0.491 & 0.416 \\
\hline & $\mathrm{p}$ & - & - & $<0.001 *$ & $0.001 *$ & $0.004^{*}$ \\
\hline
\end{tabular}

*Significant correlation, $r$ : regression coefficient.

BMI: body mass index, EFT: epicardial fat thickness, FM: fat mass, HOMA-IR: homeostasis model assessment of insulin resistance, SA: short axis view, PHFT: perihepatic fat thickness, WC: waist circumference

Table 5. ROC curves for the traditional cardiometabolic risk factors, serum chemerin, epicardial fat thickness and perihepatic fat thickness in the diagnosis of metabolic syndrome in girls with Turner syndrome

\begin{tabular}{|c|c|c|c|c|c|c|c|c|}
\hline & AUC & $95 \% \mathrm{CI}$ & Cut-off & Sensitivity (\%) & Specificity (\%) & PPV (\%) & NPV (\%) & Accuracy (\%) \\
\hline BMI Z-score & 0.998 & $0.99-1.0$ & 1.97 & 92.3 & 98 & 92.3 & 95.8 & 94.6 \\
\hline WC (cm) & 0.955 & $0.905-1.0$ & 76.50 & 92.0 & 90.8 & 75 & 95.2 & 86.4 \\
\hline HOMA-IR & 0.899 & $0.819-0.979$ & 3.32 & 91.5 & 77.6 & 66.7 & 92.8 & 81.1 \\
\hline Triglycerides (mg/dL) & 0.885 & $0.799-0.972$ & 94.0 & 92.1 & 69.4 & 63.1 & 92.8 & 78.4 \\
\hline Chemerin (ng/mL) & 0.834 & $0.715-0.952$ & 250.1 & 76.9 & 77.6 & 62.5 & 85.7 & 75.6 \\
\hline EFT-SA mDixon (mm) & 0.814 & $0.677-0.951$ & 6.20 & 84.6 & 73.5 & 64.7 & 90 & 78.4 \\
\hline EFT-SA systole (mm) & 0.800 & $0.662-0.938$ & 6.15 & 83.8 & 63.3 & 55 & 88.2 & 70.3 \\
\hline EFT-SA diastole (mm) & 0.807 & $0.681-0.933$ & 3.55 & 80.9 & 71.4 & 61.1 & 89.4 & 75.7 \\
\hline PHFT (mm) & 0.685 & $0.502-0.868$ & 5.15 & 72.7 & 57.1 & 50 & 82.3 & 64.8 \\
\hline
\end{tabular}


Informed Consent: Informed consent was obtained from the parents of all participants included in the study.

\section{Peer-review: Externally peer-reviewed.}

\section{Authorship Contributions}

Concept: Nanees A. Salem, Nihal M. Batouty, Wafaa Laimon, Design: Nanees A. Salem, Nihal M. Batouty, Wafaa Laimon, Data Collection or Processing: Nanees A. Salem, Nihal M. Batouty, Wafaa Laimon, Analysis or Interpretation: Nanees A. Salem, Nihal M. Batouty, Ahmed M. Tawfik, Donia M. Sobh, Basma Gadelhak, Shimaa R. Hendawy, Wafaa Laimon, Literature Search: Nanees A. Salem, Nihal M. Batouty, Ahmed M. Tawfik, Donia M. Sobh, Basma Gadelhak, Shimaa R. Hendawy, Wafaa Laimon, Writing: Nanees A. Salem, Nihal M. Batouty.

Financial Disclosure: The authors declared that this study received no financial support.

\section{References}

1. Skinner AC, Ravanbakht SN, Skelton JA, Perrin EM, Armstrong SC. Prevalence of obesity and severe obesity in US children, 19992016. Pediatrics. 2018;141:e20173459. Erratum in: Pediatrics 2018;142:e20181916. 2018 Feb 26.

2. Magge SN, Goodman E, Armstrong SC; Committee on Nutrition; Section on Endocrinology; Section on Obesity. The metabolic syndrome in children and adolescents: shifting the focus to cardiometabolic risk factor clustering. Pediatrics 2017;140:e20171603.

3. Gravholt $\mathrm{CH}$, Andersen NH, Conway GS, Dekkers OM, Geffner ME, Klein KO, de Vries L, Tenenbaum A, Phillip M, Lazar L; International Turner Syndrome Consensus Group. Clinical practice guidelines for the care of girls and women with Turner syndrome: proceedings from the 2016 Cincinnati International Turner Syndrome Meeting. Eur J Endocrinol 2017;177:G1-G70.

4. Gawlik A, Gieburowska J, Malecka-Tendera E. Czynniki ryzyka sercowometabolicznego w zespole Turnera [Cardiometabolic risk factors in Turner syndrome]. Pediatr Endocrinol Diabetes Metab 2015;20:69-74.

5. O'Gorman CS, Syme C, Lang J, Bradley TJ, Wells GD, Hamilton JK. An evaluation of early cardiometabolic risk factors in children and adolescents with Turner syndrome. Clin Endocrinol (Oxf) 2013;78:907913. Epub 2013 Apr 6

6. Mazzanti L, Bergamaschi R, Castiglioni L, Zappulla F, Pirazzoli P, Cicognani A. Turner syndrome, insulin sensitivity and growth hormone treatment. Horm Res 2005;64(Suppl 3):51-57. Epub 2006 Jan 20

7. Lebenthal Y, Levy S, Sofrin-Drucker E, Nagelberg N, Weintrob N, Shalitin S, de Vries L, Tenenbaum A, Phillip M, Lazar L. The natural history of metabolic comorbidities in turner syndrome from childhood to early adulthood: comparison between $45, \mathrm{x}$ monosomy and other karyotypes. Front Endocrinol (Lausanne) 2018;9:27.

8. Gray SL, Vidal-Puig AJ. Adipose tissue expandability in the maintenance of metabolic homeostasis. Nutr Rev 2007;65:S7-S12

9. Neeland IJ, Ross R, Després JP, Matsuzawa Y, Yamashita S, Shai I, Seidell J, Magni P, Santos RD, Arsenault B, Cuevas A, Hu FB, Griffin B, Zambon A, Barter P, Fruchart JC, Eckel RH; International Atherosclerosis Society; International Chair on Cardiometabolic Risk Working Group on Visceral Obesity. Visceral and ectopic fat, atherosclerosis, and cardiometabolic disease: a position statement. Lancet Diabetes Endocrinol 2019;7:715725. Epub 2019 Jul 10

10. Bertaso AG, Bertol D, Duncan BB, Foppa M. Epicardial fat: definition, measurements and systematic review of main outcomes. Arq Bras Cardiol 2013;101:e18-e28.

11. Nagy E, Jermendy AL, Merkely B, Maurovich-Horvat P. Clinical importance of epicardial adipose tissue. Arch Med Sci 2017;13:864874. Epub 2016 Oct 26

12. Fitzgibbons TP, Czech MP. Epicardial and perivascular adipose tissues and their influence on cardiovascular disease: basic mechanisms and clinical associations. J Am Heart Assoc 2014;3:e000582.

13. Iacobellis G, Willens HJ. Echocardiographic epicardial fat: a review of research and clinical applications. J Am Soc Echocardiogr 2009;22:13111319; quiz 1417-1418.

14. Goralski KB, McCarthy TC, Hanniman EA, Zabel BA, Butcher EC, Parlee SD, Muruganandan S, Sinal CJ. Chemerin, a novel adipokine that regulates adipogenesis and adipocyte metabolism. J Biol Chem 2007;282:28175-28188. Epub 2007 Jul 16

15. Lehrke M, Becker A, Greif M, Stark R, Laubender RP, von Ziegler F, Lebherz C, Tittus J, Reiser M, Becker C, Göke B, Leber AW, Parhofer KG, Broedl UC. Chemerin is associated with markers of inflammation and components of the metabolic syndrome but does not predict coronary atherosclerosis. Eur J Endocrinol 2009;161:339-344. Epub 2009 Jun 4

16. Wu Q, Chen Y, Chen S, Wu X, Nong W. Correlation between adiponectin, chemerin, vascular endothelial growth factor and epicardial fat volume in patients with coronary artery disease. Exp Ther Med 2020;19:10951102. Epub 2019 Dec 5

17. Kaur J, Mattu HS, Chatha K, Randeva HS. Chemerin in human cardiovascular disease. Vascul Pharmacol 2018;110:1-6. Epub 2018 Aug 3

18. Ghalli I, Salah N, Hussien F, Erfan M, El-Ruby M, Mazen I, et al. Egyptian growth curves for infants, children and adolescents 2002. In: Satorio A, Buckler JMH, Marazzi N (eds). Italy: Ferring Publisher; 2008.

19. Bonthuis M, Jager KJ, Abu-Hanna A, Verrina E, Schaefer F, van Stralen $\mathrm{KJ}$. Application of body mass index according to height-age in short and tall children. PLoS One 2013;8:e72068.

20. de Onis M, Onyango AW, Borghi E, Siyam A, Nishida C, Siekmann J. Development of a WHO growth reference for school-aged children and adolescents. Bull World Health Organ 2007;85:660-667.

21. Flynn JT, Kaelber DC, Baker-Smith CM, Blowey D, Carroll AE, Daniels SR, de Ferranti SD, Dionne JM, Falkner B, Flinn SK, Gidding SS, Goodwin C, Leu MG, Powers ME, Rea C, Samuels J, Simasek M, Thaker VV, Urbina EM; Subcommittee on Screening and Management of High Blood Pressure in Children. Clinical practice guideline for screening and management of high blood pressure in children and adolescents. Pediatrics 2017;140:e20171904. Erratum in: Pediatrics 2017. Erratum in: Pediatrics 2018;142. Epub 2017 Aug 21

22. Calcaterra V, Brambilla P, Maffè GC, Klersy C, Albertini R, Introzzi F, Bozzola E, Bozzola M, Larizza D. Metabolic syndrome in Turner syndrome and relation between body composition and clinical, genetic, and ultrasonographic characteristics. Metab Syndr Relat Disord 2014;12:159-164. Epub 2014 Jan 21

23. Álvarez-Nava F, Racines M, Witt J, Guarderas J, Estévez M, Lanes R. Anthropometric variables as cardiovascular risk predictors in a cohort of adult subjects with Turner syndrome. Diabetes Metab Syndr Obes 2019;12:1795-1809.

24. Kim SJ, Kim HS, Jung JW, Kim NS, Noh CI, Hong YM. Correlation between epicardial fat thickness by echocardiography and other 
parameters in obese adolescents. Korean Circ J 2012;42:471-478. Epub 2012 Jul 26.

25. Akyol B, Boyraz M, Aysoy C. Relationship of epicardial adipose tissue thickness with early indicators of atherosclerosis and cardiac functional changes in obese adolescents with metabolic syndrome. J Clin Res Pediatr Endocrinol 2013;5:156-163.

26. López-Bermejo A, Prats-Puig A, Osiniri I, Martínez-Calcerrada JM, Bassols J. Perirenal and epicardial fat and their association with carotid intima-media thickness in children. Ann Pediatr Endocrinol Metab 2019;24:220-225. Epub 2019 Dec 31

27. Ostberg JE, Thomas EL, Hamilton G, Attar MJ, Bell JD, Conway GS. Excess visceral and hepatic adipose tissue in Turner syndrome determined by magnetic resonance imaging: estrogen deficiency associated with hepatic adipose content. J Clin Endocrinol Metab 2005;90:2631-2635. Epub 2005 Feb 15.
28. Abaci A, Tascilar ME, Saritas T, Yozgat Y, Yesilkaya E, Kilic A, Okutan $\mathrm{V}$, Lenk MK. Threshold value of subepicardial adipose tissue to detect insulin resistance in obese children. Int J Obes (Lond) 2009;33:440446. Epub 2009 Feb 17

29. Okyay K, Balcioglu AS, Tavil Y, Attar MJ, Bell JD, Conway GS. A relationship between echocardiographic subepicardial adipose tissue and metabolic syndrome. Int J Cardiovasc Imaging 2008;24:577-583. Epub 2008 Jan 26.

30. Thomas EL, Frost G, Taylor-Robinson SD, Bell JD. Excess body fat in obese and normal-weight subjects. Nutr Res Rev 2012;25:150-161.

31. Zhang Y, Chen RM, Lin XQ, Yuan X, Yang XH. The correlation between serum adipokines levels and metabolic indicators in girls with Turner syndrome. Cytokine 2019;113:139-143.

Click for Supplementary Figure 1, Supplementary Table 1 and Table 2 access link: http://glns.co/6gg7o 